\title{
Subspecialty certification in urology: a European perspective
}

Frans MJ Debruyne

'Superspecialization', the certification of subspecialties, is an important issue in the future development of urology. Whereas, in the early years, urologists tended to be urogenital surgeons, today it is impossible to master all aspects of urology or to be experienced in all types of urologic surgery. There are a number of challenges relating to the superspecialization of urology in Europe. Firstly, Europe consists of 50 separate countries with many regional and national urology associations. The current status of superspecialization also differs between countries and regions. In Germany, for example, there is already an established difference between office urology and operative clinical urology. This general differentiation, however, does not exist in most of the other European countries where urologists are not restricted in what diagnostic and therapeutic approaches they can employ.

The opportunities for superspecialization also differ between regions of Europe, in particular between Eastern, Western, and central Europe. Generally speaking, facilities are less developed in Eastern European countries compared to those in central Europe. Central European countries are rapidly attaining the facilities that are currently available in Western Europe, and so over time these differences should be less apparent.

In Europe, there is no regulation of superspecialization and, therefore, no official accreditation is available. The urology section of the regulatory body for the organizational division of different medical specialties in the European Union (The Union Européenne des Médecins Spécialistes), has not yet produced a document stating the need for superspecialization or the qualifications required for the certification of a urologic superspecialist. Despite this, the arguments for superspecialization in urology are compelling. Across the various national societies and Pan-European professional scientific organizations, such as the European Association of Urology, it is generally felt that there is a need for superspecialization. Some super-

specialization

in urology....is

essential. .. . [it]

is also crucial

to future

developments

within the

specialty

FMJ Debruyne

is Professor of

Urology at Radboud

University Nijmegen

Medical Centre, The

Netherlands.

Competing interests

The author declared he has

no competing interests.

www.nature.com/clinicalpractice doi:10.1038/ncpuro0656 national societies have even started in-depth discussions. The process of regulating superspecialization in Europe will take time, and it will probably be over a decade before a consensus is reached across all European countries.

Urology can be divided into five superspecialist fields: minimally invasive urology, andrologic urology, female and functional urology, oncologic urology and pediatric urology. Each of these fields has problems that need to be overcome before they can become superspecialties. Establishing minimally invasive surgery as a superspecialty could be particularly difficult because of the difference in facilities available across Europe. The prevailing opinion, particularly among junior urologists, is that all urologists should practice laparoscopy. Before this can be achieved, however, training and equipment availability will need to be regulated. The future urology superspecialties can only be established within a multidisciplinary framework. This is only partially true for pediatric urology, although, in many European countries, pediatric surgeons have taken responsibility for the care of some pediatric urology patients. For the future of urology, a multidisciplinary approach is essential, in order to improve diagnosis with the use of imaging techniques and novel molecular tests, and in order to offer patients the full range of treatments available.

In order for superspecialization to be accepted, organized and certified in Europe, urologists will need to be willing to integrate it into their daily practice. Superspecialization will differentiate between surgically active urologists and those involved in diagnosis and medical treatment. It will automatically differentiate between office urology and operative clinical urology and, at the same time, will lead to further developments in multidisciplinary approaches in urology. The need for superspecialization in urology, and its regulation and certification, is essential. This is not only a consequence of progress in urology, but is also crucial to future developments within the specialty. 